\title{
Local Anesthetic Spread to the Contralateral Trigeminal Ganglion
}

\author{
Kim T. Nguyen, MD, Ariana Anugerah, MD, and Antoun Nader, MD
}

Chronic neuropathic pain syndromes can severely affect a person's quality of life. Trigeminal neuralgia is among these syndromes and involves the mandibular branch of the fifth cranial trigeminal nerve. Patients typically present with facial pain described as electrical and shock-like in nature, exacerbated by movements of the jaw such as talking and chewing. Many patients are unable to achieve adequate relief with medical management and therefore require interventions such as injections of local anesthetics, steroids, or glycerol. One method of injection uses ultrasound guidance for local anesthetic injection in the pterygopalatine fossa affecting the trigeminal ganglion.

A 64-year-old woman with a history of trigeminal neuralgia presented for a trigeminal nerve block on her right side via ultrasound guidance. Following the injection, she experienced the desired effect of numbness on her right side in the distribution of the trigeminal nerve. In addition, the patient reported feeling the same effects on her left side despite no injection being done on that side.

We describe a unique case in which local anesthetics spread through the pterygopalatine fossa and across the midline, affecting the contralateral trigeminal ganglion. Patients should be monitored for hemodynamic changes following this injection.

Key words: Trigeminal neuralgia, trigeminal nerve block, pterygopalatine fossa, regional block, trigeminal ganglion
Trigeminal neuralgia is a chronic neuropathic pain syndrome that involves the fifth cranial nerve, primarily the maxillary branch (V2) mandibular branch (V3) (1). It is also known as tic douloureux or suicide disease and can severely affect a person's quality of life (2). It is the most common form of neuralgia in adults and is seen in as many as 5 per 100,000 (3). Trigeminal neuralgia is more commonly seen in women over the age of 40 (4). This syndrome is typically described as a sudden, unilateral, electrical,

From : Northwestern University Feinberg School of Medicine, Department of Anesthesiology

Author for correspondence: Kim T. Nguyen, MD

Address: Northwestern University Feinberg School of Medicine, Department of Anesthesiology, 251 E. Huron, F5-704, Chicago, IL 60611 E-mail: kim.nguyen@northwestern.edu shock-like and intractable pain in the face (1). This sensation lasts anywhere from a few seconds to as long as minutes to hours and could involve a series of attacks. Symptoms can be triggered by movements involved in talking, chewing, or brushing teeth. In some instances, symptoms are triggered by just light touch or air currents to the face. Atypically, the syndrome may appear as a persistent facial pain.

Medical management with carbamazepine or oxcarbazepine is first-line treatment. Approximately $25 \%$ of patients require further intervention for pain management (3). Options include injections, radiofrequency treatments, balloon decompression of the trigeminal ganglion, gamma-knife radiation, or other surgical procedures (5). Injections are typically done under image guidance with fluoroscopy, computed tomography, or ultrasound. Ultrasound guidance allows for real-time visualization of soft tissues, surround vasculature, and bony structures. It allows for the fine 
adjustment of the needle tip and direct observation of injectate spread. For the trigeminal nerve block, the injectate is injected into the pterygopalatine fossa affecting the sphenopalatine ganglion, superficial and deep petrosal nerves, and trigeminal ganglion (3) (Fig. 1). The result is sensory analgesia or a numbness sensation in the distribution of the trigeminal nerve. In addition, facial droop may also be seen due to the close proximity of the motor nucleus of the facial nerve and the trigeminal sensory nuclei (3). We present the case of a patient who experienced the desired effects of a trigeminal nerve block on the indicated side but also described numbness on her contralateral side.

\section{CASE}

A 64-year-old woman with a history of trigeminal neuralgia presented for a trigeminal nerve block for continued management at the chronic pain clinic. The ultrasound probe was placed below the zygomatic bone, superior to the mandibular notch, and anterior to the mandibular condyle (Fig. 2). The pterygopalatine fossa was then identified anterior to the lateral pterygoid plate and below the lateral pterygoid muscle (6). An injection of $5 \mathrm{~mL}$ of $0.5 \%$ bupivacaine and 40 $\mathrm{mg}$ of triamcinolone acetonide into the fossa was performed on the patient's right side. Following the injection, the patient reported the desired effects of right-sided numbness in the distribution of the trigemi-

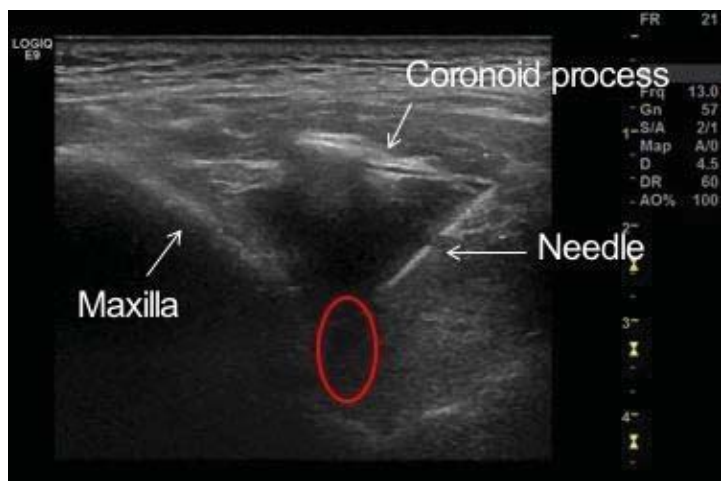

Fig. 1. Ultrasound image showing the needle in the pterygopalatine fissure (red). The ultrasound probe is placed below the zygomatic bone, superior to the mandibular notch, and between the coronoid process and the mandibular condyle. nal nerve as well as facial drooping. In addition, the patient reported numbness in her left side despite no intervention to that side.

\section{DISCUSSION}

It has been shown that patients can achieve pain relief from trigeminal neuralgia with a trigeminal nerve block using ultrasound guidance (3). Previously, images have been taken using fluoroscopy and contrast dye injected via ultrasound guidance as described above. These images demonstrated the injectate traveling from the pterygopalatine fossa proximally through the foramen rotundum (3). The dye traveled to Meckel's cave, where the trigeminal ganglion (or Gasserian ganglion) lies within the middle cranial fossa (3). Furthermore, this previous study showed that the contrast dye continued to flow retrograde to the anterior surface of the brainstem, allowing the injectate to travel to the contralateral side (Fig. 3). Additionally, in cadaveric specimens, methylene blue was seen within the pterygopalatine fossa (7). This resulted in staining of the pterygopalatine ganglion, maxillary nerve, greater and lesser palatine nerves, and the superior alveolar nerves (7).

The pterygopalatine fossa is very small and only 2 $\mathrm{mL}$ of contrast dye was needed to demonstrate retrograde flow to the anterior brainstem (3). This patient received $6 \mathrm{~mL}$ of injectate, making retrograde flow not

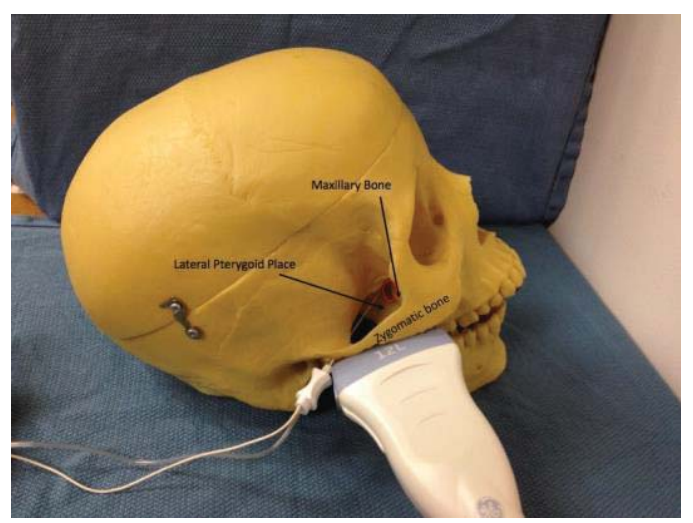

Fig. 2. The ultrasound transducer is placed below the zygomatic bone. The pterygopalatine fossa (red) is anterior to the lateral pterygoid plate. 
only reasonable, but this also explains the symptoms the patient experienced on her contralateral face.

There were a few concerns given the patient's symptoms and spread of local anesthetics to the anterior brainstem. If local anesthetics were to significantly affect the ganglion, other cranial nerves, and the surrounding structures, this could lead to concerns for hemodynamic instability and airway compromise. More severely, total central neurologic block or total spinal anesthesia could be a major consequence. This would be associated with apnea, fixed dilated pupils, hypotension, and loss of consciousness. Although we suggest that local anesthetics traveling to the anterior brainstem caused the patient's symptoms, we believe it is unlikely that a patient would experience a high or total spinal. Previous studies have shown that high or total spinals are usually a result of spinal blocks, epidurals, and interscalene blocks (8). These procedures involve varying concentrations, volumes, and locations of injection compared to the trigeminal nerve block. Furthermore, the technique we described has been performed in over 1000 cases with no reported side effects. Regardless, although our patient did not show any signs of hemodynamic compromise, this case demonstrates the importance of hemodynamic monitoring for at least 30 minutes after the injection.

This is the only known case of retrograde flow of local anesthetic during a trigeminal nerve block in the literature. The anatomical course of the trigeminal nerve makes it conceivable that the local anesthetic was able to reach the anterior surface of the brainstem and therefore affect the contralateral side.

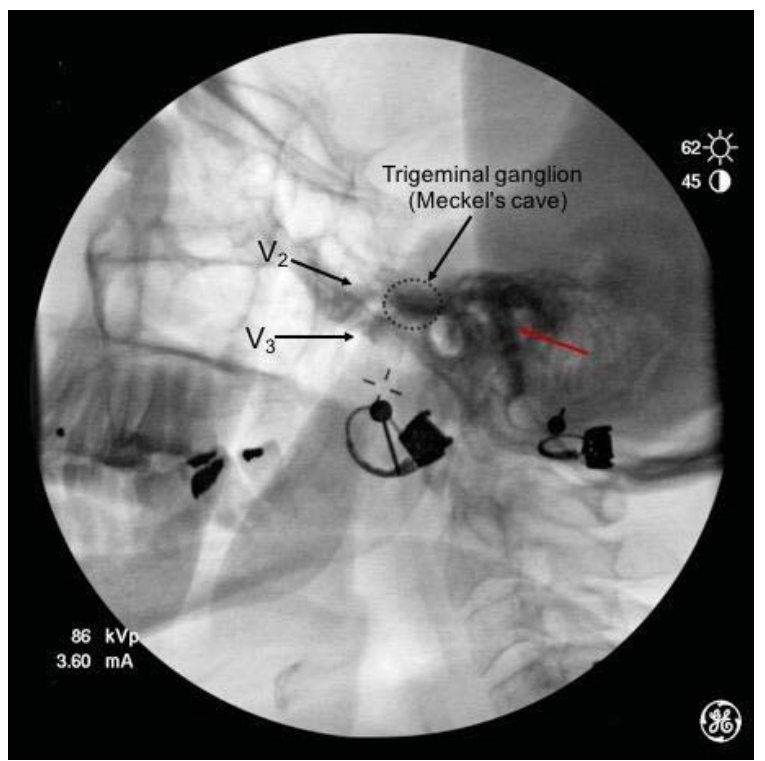

Fig. 3. Using fluoroscopy, it has been shown that contrast dye injected into the pterygopalatine fossa can spread retrograde to the middle cranial fossa and the brain stem (red arrow).

In conclusion, we present a case in which the patient experienced numbness following a trigeminal nerve block in both the desired and contralateral sides of her face. This is likely due to retrograde local anesthetic flow to the anterior surface of the brainstem, allowing it to travel to the contralateral side. Although the patient did not have any hemodynamic changes as a result of local anesthetic spread, this case suggests the need for hemodynamic monitoring following a trigeminal nerve block. 


\section{REFERENCES}

1. Cruccu G, Biasiotta A, Galeotti F, lannetti GD, Truini A, Gronseth G. Diagnostic accuracy of trigeminal reflex testing in trigeminal neuralgia. Neurology 2006; 66:139-141.

2. Cruccu G. Trigeminal neuralgia. Continuum (Minneap Minn) 2017; 23:396-420.

3. Nader A, Kendall MC, De Oliveria GS, Chen JQ, Vanderby B, Rosenow JM, Bendok BR. Ultrasound-guided trigeminal nerve block via the pterygopalatine fossa: An effective treatment for trigeminal neuralgia and atypical facial pain. Pain Physician 2013; 16:537-545.

4. Merskey H, Bogduk N; International Association for the Study of Pain, eds. \{AU: Is IASP meant to be author, editor, or part of your title? Original citation unclear.\} Classification of Chronic Pain: Descriptions of Chronic Pain Syndromes and Definitions of Pain Terms. 2nd ed. International Association for the Study of Pain Press, Seattle, WA, 1994.

5. Dalessio DJ. Trigeminal neuralgia. A practical approach to treatment. Drugs 1982; 24:248-255.

6. Nader A, Schittek H, Kendall MC. Lateral pterygoid muscle and maxillary artery are key anatomical landmarks for ultrasoundguided trigeminal nerve block. Anesthesiology 2013; 118:957.

7. Kampitak W, Tansatit T, Shibata Y. A cadaveric study of ultrasound-guided maxillary nerve block via the pterygopalatine fossa: A novel technique using the lateral pterygoid plate approach. Reg Anesth Pain Med 2018; 43:625-630.

8. Park P, Berry P, Larson M. Total spinal anesthesia following epidural saline injection after prolonged epidural anesthesia. Anesthesiology 1998; 89:1267-1270. 